\title{
Insurance Denials of NICU Hospital Claims
}

\section{Eugene L. Mahmoud, MD}

With regards to the defined principles of Medical Necessity and Standards of Medical Care, there have been significant differences among those who are primarily involved in patient care among patients, physicians, insurance payers, and hospital administrations. In general, the concepts of experimental care and investigational care are concluded to be the opposite of Medical Necessity and Standards of Care. As it relates to Neonatology, preterm birth and low birthweight birth exert significant, medical, social, and economic costs on affected families and the United States healthcare system. Preterm birth is the leading cause of neonatal mortality and is a significant cause of both short and long-term morbidity and disability. While the least mature newborns have the highest average per individual medical expenditures, data from 2017 showed newborns born at 26 weeks gestational age represent $4 \%$ of all preterm birth with the medical expenditure of $22.5 \%$. But the more mature infants (at 33-36 weeks gestational age) as a cohort representing approximately $80 \%$ of all preterm births have higher total expenditures at $38 \%$. In addition, these data do not include mature term gestation newborns with complex congenital heart conditions or other complex conditions noted at birth.

\section{"The physician's decision of when to discharge an infant from the hospital after a stay in the Neonatal Intensive Care Unit (NICU) is complex."}

The physician's decision of when to discharge an infant from the hospital after a stay in the Neonatal Intensive Care Unit (NICU) is complex. This decision is made primarily on the basis of the infant's medical status, and whether the specifically indicated care needs may only be accomplished at the acute hospital level of care or if the care needs may be achieved at a lower level of care - Normal Nursery, a rehabilitation facility, or care at home with care by parents and other health care professionals (nurses, therapists). Also, NICU Hospital Discharge is complicated by the readiness of families for discharge and pressures to contain hospital costs by shortening the length of stay. Insofar as possible, the determination of the readiness for discharge should be based on current peerreviewed scientific evidence. Historically, preterm infants were discharged only when they achieved a certain weight, typically 2250 grams (5 pounds). Currently, randomized clinical trials have shown that earlier discharge is possible without adverse health effects when preterm infants are discharged on the basis of physiologic criteria rather than body weight. The three physiologic competencies that are generally recognized as essential before hospital discharge of the preterm infant are 1. Oral feeding sufficient to support appropriate growth. 2 . The ability to maintain a normal body temperature in a home environment. 3. Sufficient mature respiratory control. These competencies are achieved by most preterm infants between 36 and 37 weeks' postmenstrual age. However, maturation to the point that allows safe discharge may take longer, occasionally up to 44 weeks' postmenstrual age. Although interrelated, not all competencies are achieved by the same postnatal age in a given infant. The pace of maturation is influenced by the birth weight, the gestational age at birth, and the degree and chro- nicity of neonatal illnesses. Infants born earlier in gestation and with more complicated medical courses tend to take longer to achieve these physiologic competencies.

The American Academy of Pediatrics (AAP) Private Payer Advocacy Advisory Committee (PPAAC) identifies opportunities to engage private payers on pediatric issues, coverage and payment within the limits of antitrust regulations http://bit.ly/AAPHassleFactorForm. Several AAP chapters also have formed pediatric councils to meet with local payers on pediatric issues.

To ensure that carriers consider the unique aspects of pediatrics when making coverage determinations, the Academy has implemented a process with Anthem, United Health Corporation, and the Blue Cross Blue Shield Association in which pediatricians review medical policies that impact children. Such a review does not imply AAP endorsement of a carrier's policy but advises the carrier on how the policy impacts pediatrics and pediatricians and advocates for appropriate benefits coverage.

"To ensure that carriers consider the unique aspects of pediatrics when making coverage determinations, the Academy has implemented a process with Anthem, United Health Corporation, and the Blue Cross Blue Shield Association in which pediatricians review
medical policies that impact children."

A study by the Government Accountability Office found that many denials can be traced to largely trivial bureaucratic issues, such as a missing form or an incorrect billing code. The study found that when patients challenged the insurers' denials, about half of the rejected claims ended up being covered.

Insurers employ warehouses full of claims adjusters who, as a primary function, scrutinize claims for pretexts of lack of documentation to deny care, saying it is 'experimental,' 'must abide by third party insurance decision guidelines,' or 'not medically necessary' even when the medical treatment, prescription medication, diagnostic procedure or referral to a specialist is recommended by doctors.

Informed health care leads to the best patient outcomes, avoid-

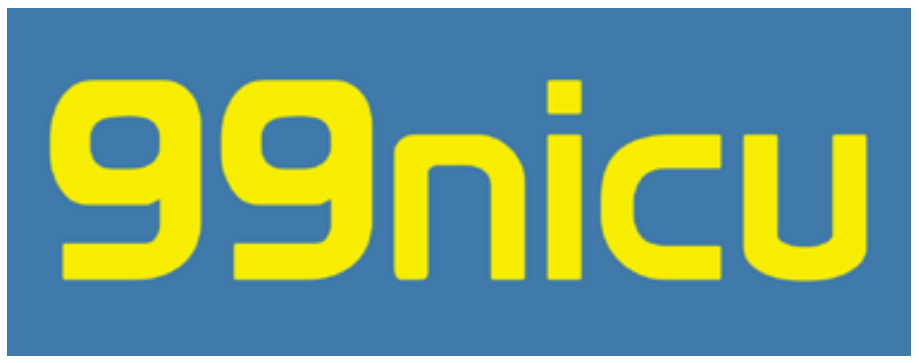


ing underuse or overuse of medical resources. Evidence-based care guidelines from Milliman Care Guidelines and InterQual Guidelines help providers, and health plans drive informed care in their own work and through the conversations that connect them. Sometimes, there can be confusion when some insurance carriers adopt Milliman Care Guidelines, and others adopt InterQual Guidelines. Because when the two guidelines do not match exactly, the approval for the NICU level of care can be difficult to interpret. Each care guideline recognizes the four NICU levels of care, which include prematurity, severe conditions, mild instabilities, and routine newborn care. However, high flow nasal cannula systems (HFNC) deliver oxygen via a system that heats, humidifies, and then delivers a $24-100 \%$ (0.24-1.0) $\mathrm{ViO} 2$ at body temperature through nasal cannula prongs, HFNC is used to provide a high flow without causing barotrauma. It appears to have similar clinical efficacy and safety to nasal continuous positive airway pressure (CPAP) as a mode of noninvasive respiratory support. For infants up to 12 months of age, HFNC may be administered at rates as high five (5) liters per minute (LPM). So, some insurance carriers may accept HFNC for the highest level of medically necessary care who adopt the InterQual Guidelines, when those insurance carriers who adopt the only the Milliman Care Guidelines may not accept HFNC for newborns requiring the highest level of medically necessary care. As the Milliman Care Guideline is frequently updated, it is hoped that newborns receiving HFNC will be included with those at the highest levels of NICU care.

"So, some insurance carriers may accept HFNC for the highest level of medically necessary care who adopt the InterQual Guidelines, when those insurance carriers who adopt the only the Milliman Care Guidelines may not accept HFNC for newborns requiring the highest level of medically necessary care."

For lack of documentation and coding of health care, the insurers are justified to deny care. However, these are rare circumstances. When taking care of extremely sick patients in the NICU and Pediatric Intensive Care Unit (PICU) meticulous documenting the complexity of care is reflected in detailed progress reports and input from specialist consultation. Medical necessity means health care services that a physician, exercising prudent clinical judgment, would provide to a patient. The service must be for the purpose of evaluating, diagnosing, or treating an illness, injury, disease, or its symptoms. In the treatment of severely ill patients, the physicians employ therapy based on current on peer-reviewed scientific evidence with the Food and Drug Administration (FDA) support in accordance with the standard of care.

By means of careful preparation, Neonatologists can minimize potential audits and defend against inappropriate payer denials and repayment demands. Since the introduction of health maintenance organizations (HMOs) and other managed care organizations that perform utilization review, there has been public concern that decisions about insurance coverage for diagnostic and therapeutic services might be based on the cost to the insurer rather than the clinical appropriateness. HMOs since 1975 are required to provide an internal grievance process, in which an enrollee who was denied coverage of the desired treatment could appeal the decision. In response to additional appeals to health care organizations, the HMOs insurer payers provide an additional external independent medical review (IRO) for coverage denials. In the external review process, health plans, patients, providers, and medical institutions take their disagreements to a regulatory board. Within this process, requests for the involvement of reviewing physicians with the same qualifications as those caring for the patient in question aids in getting a just resolution.

\section{References:}

1. Policy Statement: Hospital discharge of the high-risk neonate. Pediatrics. 2008;122(5):1119-1126. Available at: http://pediatrics.aappublications.org/content/122/5/1119. Reaffirmed November 2018

2. Heated, Humidified High-Flow Nasal Cannula Versus Nasal CPAP for Respiratory Support in Neonates Yoder BA, Stoddard RA, Li M, King J, Dirnberger DR, Abbas Si Pediatrics, May 2013, 131 (5) e1482-e1490

3. Estimates of Healthcare Spending for Preterm and Lowbirthweight Infants in a Commercially Insured Population: 2008-2016 Beam AL, Fried I, Palmer N, Agniel D, Brat G, Fox K, Kohane I, Sinaiko A, Zupancic AF, Armstrong J Journal of Perinatology (2020)

4. Independent Medical Review of Health Plan Coverage Denials: Early Trends Chuang K, Aubry WM, Adams Dudley $R$ Health Affairs Volume 23, Number 6, pp. 163-169

5. Subspecialists Benefit from AAP Private Payer Policy Lander et al., AAP News, 2017

6. AAP continues to resolve issues with health insurance companies Kressly SA, AAP News, 2019

7. Milliman Care Guidelines MCGTM General Recovery Care 17th Edition Introduction to Neonatal Levels of Care

8. InterQual 2017 Acute Pediatric Criteria- Nursery

9. Guide to Clinical Practice Guidelines: the Current State of Play Kredo T, Berhardsson S, Shingai Machingaidze, Young T, Louw Q, Ochodo E, and Grimmer K Int J Qual Health Care. 2016 Feb; 28(1): 122-128.

10. Miles, A., Loughlin M. Models in the balance: evidencebased medicine versus evidence-informed individuals

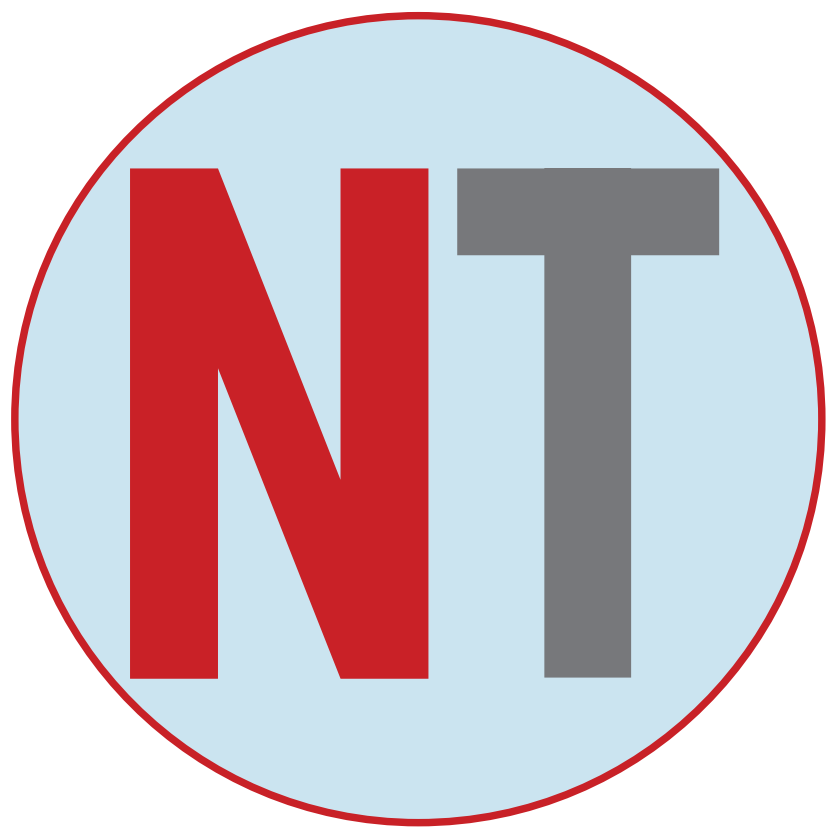


care. Journal of Evaluation in Clinical Practice (2011) 17(4): $531-536$

The author has no conflicts of interests to disclose.

NT

Corresponding Author:

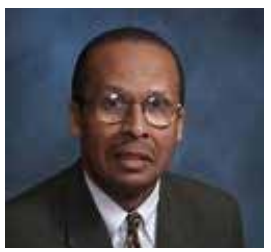

Eugene L. Mahmoud, MD

Medical Reviewer- American Health Health Holding, Advanced Medical Reviews, Dane Street, Medical Board of California Past Staff Neonatologist- UCI Medical Center, Orange, CA and Parkview Hospital Medical Center, Riverside, CA

Cell: (949)-683-0672

Fax: (626)-365-1344

Email: emahmoudsr@yahoo.com or eugene.mahmoud@gmail.com

Mailing address- 360 South Los Robles Avenue, Unit\#- 11, Pasadena, CA 91101

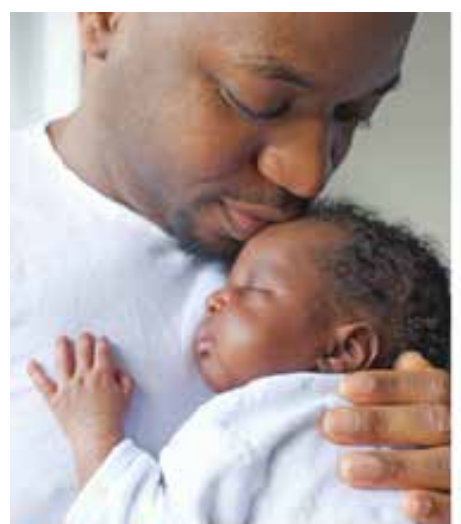

Did you know that

Join OP NPA

nationalperinatal_org/mental_health

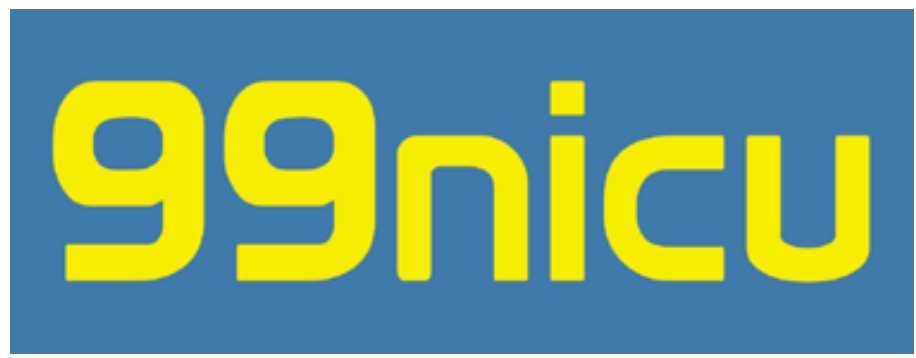
related suicides account for

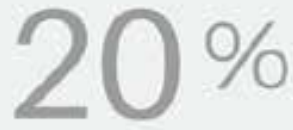

\section{of Postpartum}

Maternal Deaths?
Why PREMATURE INFANTS Need Access

to an EXCLUSIVE HUMAN MILK DIET

क्षेत है

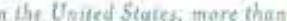
1 IN 10 BABIES ARE BORN PREMATURE

Very tea birthaceight tabien are born ieverely permieturt, avighing Ien then 8,250 grmou.
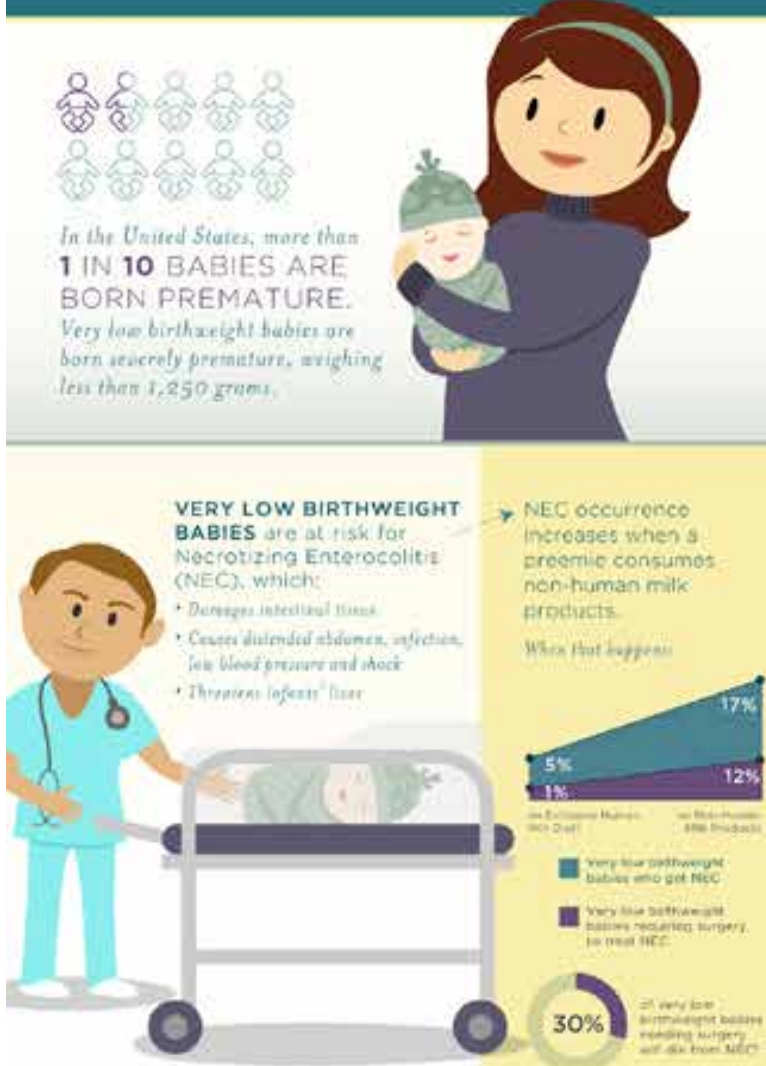

HOW TO HELP PREVENT NEC: EXCLUSIVE HUMAN MILK DIET

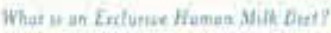
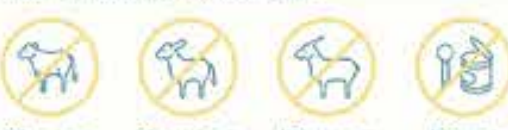

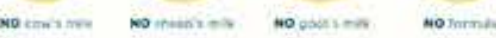

Why Is An Exclusive Human Milk Dlet incottan?

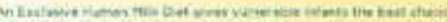

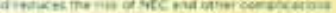

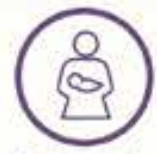

$\checkmark$ mothersma $\checkmark$ tiumian donor netia

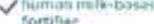

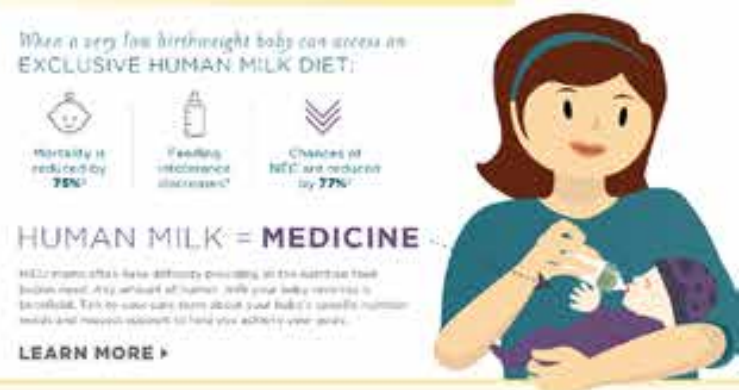

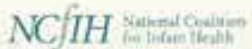

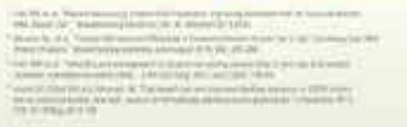

